\title{
A level set method for segmentation of the thalamus and its nuclei in DT-MRI
}

\author{
Lisa Jonasson $^{\mathrm{a}, *}$, Patric Hagmann ${ }^{\mathrm{a}, \mathrm{b}}$, Claudio Pollo ${ }^{\mathrm{a}, \mathrm{c}}$, Xavier Bresson ${ }^{\mathrm{a}}$, \\ Cecilia Richero Wilson ${ }^{\mathrm{a}}$, Reto Meuli ${ }^{\mathrm{b}}$, Jean-Philippe Thiran ${ }^{\mathrm{a}}$ \\ ${ }^{a}$ Signal Processing Institute (ITS), École Polytechnique Fédérale de Lausanne (EPFL),CH-1015 Lausanne, Switzerland \\ ${ }^{\mathrm{b}}$ Department of Diagnostic and Interventional Radiology, Lausanne University Hospital (CHUV), CH-1011 Lausanne, Switzerland \\ ${ }^{\mathrm{c}}$ Department of Neurosurgery, Lausanne University Hospital (CHUV), CH-1011 Lausanne, Switzerland
}

Received 1 March 2005; accepted 19 December 2005

Available online 23 June 2006

\begin{abstract}
We present a method for segmenting white matter as well as the gray matter structures from diffusion tensor magnetic resonance images (DT-MRI). The segmentation is done evolving a set of coupled level set functions. The zero level set of each level set function forms a surface in $3 \mathrm{D}$ that is driven by the region-based force including all tensors belonging to a certain region. The region-based force is defined by using a very sensitive similarity measure between DT. We apply our method for segmenting the thalamus and its nuclei.

This technical paper proposes several new strategies for level set methods to segment efficiently complex objects as present in DT-MRI. First of all, we present a very sensitive similarity measure that distinguishes very subtle differences between regions within, for example, the thalamus. Secondly, we present a new way of selecting the most representative tensor for group of tensors for these kinds of applications. We argue for the importance to use the tensor minimizing the variation within the group of tensors instead of the mean tensor as suggested in other papers on tensor segmentation. The third important point is the necessity of using several coupled level sets to define the background. Methods differentiating only between foreground and background will fail when applied to complex structures such as the brain. It is crucial for a region-based approach to consider all the surrounding structures for a correct definition of the forces driving the segmentation.
\end{abstract}

(C) 2006 Elsevier B.V. All rights reserved.

Keywords: Brain gray matter segmentation; Coupled level sets; DT-MRI; Thalamus; Geodesic active regions; Thalamic nuclei

\section{Introduction}

Diffusion tensor magnetic resonance imaging (DT-MRI) is a modality that permits non-invasive

\footnotetext{
*Corresponding author. Tel.: + 41216933632 ; fax: +41216937600 .

E-mail address: Lisa.Jonasson@epfl.ch (L. Jonasson).

$U R L$ : http://lswww.epfl.ch.
}

quantification of the water diffusion in living tissues. The diffusion tensor (DT) provides information about the intensity of the water diffusion in any direction at a certain point. The water diffusion in the brain is highly affected by its cellular organization. In particular, axonal cell membrane and myelin sheath are the main components restricting water mobility [1]. Hence, the measured DT becomes highly anisotropic and oriented in areas 
of compact nerve fiber organization, providing an indirect way of fiber tract identification. Today, DTMRI is mostly used for determining brain connectivity using fiber tractography algorithms [2-5]. In general, emphasis has mainly been put on identifying white matter structures, but many gray matter structures can also be revealed through this new image modality. Most gray matter structures contain passing fibers which will affect the mean diffusion within the voxel and give the DT a characteristic shape for that specific structure.

An example of such a gray matter structure is the thalamus that can be considered as the central relay station for brain neuronal communication. Every sensory system (except olfaction) makes synapses here before projecting into the cerebral cortex. Information received from diverse brain regions is passed on to the cortex through the thalamus. These passing fibers will slightly influence the anisotropy and shape of the DT. Because these axonal projections have different orientations depending on the region they connect to, diffusion is differentially oriented. This will allow us not only to segment the thalamus but also its nuclei.

The thalamus and its nuclei are structures that are hardly differentiated in other imaging modalities such as computerized tomography or conventional magnetic resonance imaging (MRI), which do not provide the necessary image contrast. Therefore, radiological identification of individual thalamic nuclei is not currently possible and even detection of the precise boundaries of the thalamus is difficult. Today the segmentation is mainly done by atlas matching or by hand segmentation procedures.

The thalamic cytoarchitecture is divided into several nuclei, each with a specific function. The thalamic nuclei have traditionally been studied with histological methods and their number varies depending on the method used. However, most studies identify 11 major nuclei, some of them being subdivided. Wiegell et al. [6] have shown how DTMRI can differentiate the principal thalamic nuclei, non-invasively, based on the characteristic fiber orientation, which is assumed to stay the same within one certain nucleus and varies from one nucleus to another. Identification of the thalamic nuclei in DT-MRI has also been done by Behrens et al. [7] by performing tractography between the thalamus and the cortex. Thalamic subregions where identified through their specific cortical connectivity.
Only recently, DT-MRI has started being used for segmentation purposes. The first approaches began by performing a fiber tractography and then used the result for segmentations [8]. Identification of the thalamic nuclei has been made by Behrens et al. [7] by mapping the connections between the thalamus and the cortex. Wiegell et al. [6] were one of the first to segment DT-MRI directly from the data by using a $k$-means algorithm. As mentioned before, this method was also used to segment the thalamic nuclei. The most recent approaches have been to use partial differential equations (PDE), variational methods and level sets [9-15]. In [9] we presented a geometric flow implemented with level set methods for fiber tract segmentation by measuring the diffusive similarity between voxels. The method is based on the assumption that adjacent voxels in a tract have similar diffusion properties but that the diffusion at different location of the tract can be very different. Similarity measures based on the whole tensor information was used for propagating the flow. Since then, several papers have provided a well developed theory on PDE and segmentation in DT-MRI [12-17]. Wang et al. [12] were the first to define regions from the DT and used region-based forces for the front propagation. The region-based force is defined from a distance metric between tensors. Wang et al. presented in [16] the very interesting approach of Kullback-Leibler (KL) distances. The KL distance is a frequently used concept in information theory and is a measure of the natural distance between two random variables. From this distance, Wang et al. [16] have derived similarity measures and segmented 2D tensor fields. Lenglet et al. [17] extended their work for $3 \mathrm{D}$ images and explored the statistics of the KL distances to segment tensor fields with higher internal variance. In this paper however, we are not interested in allowing a high internal variance, rather in detecting the very small variances that exist between the thalamic nuclei.

Our method consists in propagating a set of coupled level sets through a region-based force according to Paragios et al. [18]. The force is defined from the similarity measure between the most representative tensor of each level sets and its neighboring voxels. All surfaces evolve while remaining dependent on each other through a coupling force [18].

First, we will briefly present the concept of DT and basic theories on region-based front propagation with level set implementation. We will then 
show how to use similarity measures for DT to propagate a surface and how this can be used for white and gray matter segmentation. The results are compared to anatomical atlases.

\section{Theory}

\subsection{DT imaging and tensor similarity measures}

DT-MRI is an imaging modality that provides a second-order DT, a 3 by 3 symmetric and positive definite matrix in every voxel. Under the DT model, the shape of the diffusion probability density function in every voxel is estimated by an anisotropic Gaussian.

The diffusion coefficient in a certain direction, $\hat{x}$, where $\hat{x}$ is a unit vector in $\mathbb{R}^{3}$, is given by the double contraction of the DT with the vector:

$d(\hat{x})=\hat{x}^{T} D \hat{x}$.

A way of directly comparing the diffusion in two different voxels is to compare the diffusion coefficient over all directions, $\hat{x}$, covering the unit sphere, $S^{2}$. We define the integrated similarity (IS) between two tensors $\left(D_{1}, D_{2}\right)$ as

$\operatorname{IS}\left(D_{1}, D_{2}\right)=\frac{1}{4 \pi} \int_{S^{2}} \min \left(\frac{d_{1}(\hat{x})}{d_{2}(\hat{x})}, \frac{d_{2}(\hat{x})}{d_{1}(\hat{x})}\right) \mathrm{d} \hat{x}$,

where $d_{1}(\hat{x})$ is the diffusion in direction $\hat{x}$ for the DT $D_{1}$. The IS gives us a percentage of the common diffusion for the two tensors. Several other measures of similarity between tensors exist, see [19], but this similarity measure is particularly, sensitive to very subtle changes between tensors which is useful when separating structures such as the thalamic nuclei.

To find the most representative tensor among a group of DTs, Jones et al. [20] use a distance metric, $d_{12}$, between two tensors, $\left(D_{1}, D_{2}\right)$ :

$d_{12}:=\sqrt{\left(D_{1}-D_{2}\right):\left(D_{1}-D_{2}\right)}$,

where : stands for the tensor scalar product. This is a measure of the overlap between two tensors:

$D_{1}: D_{2}=\operatorname{Trace}\left(D_{1} D_{2}\right)=\sum_{j=1}^{3} \sum_{i=1}^{3} \lambda_{1 i} \lambda_{2 j}\left(e_{1 i} e_{2 j}\right)^{2}$,

where $\lambda_{1 i}, \lambda_{2 i}$, are the eigenvalues and $e_{1 i}, e_{2 i}$ the eigenvectors of the DT $D_{1}$ and $D_{2}$, respectively. For determining the most representative tensor of a data set, this distance is computed between each pair of tensors. Thus, for each tensor we compute $c_{i}$ :

$c_{i}=\frac{\sqrt{\sum_{i=1, j \neq i}^{n} d_{i j}^{2}}}{n-1}$,

where $n$ is the number of tensors within the region. The most representative tensor is then the tensor with lowest value of $c_{i}$.

We will use this approach later to define the most representative tensor of the set of tensors contained within our level set.

\subsection{Geometric flows and level set implementation}

Geometric flows and especially curvature or curve shortening flows are today important tools in computer vision. A curvature flow is a curve or surface that evolves at each point along the normal with the velocity depending on the curvature at that point. This process leads to a smoothing of the curves or surfaces and eliminates noise effects. The theory is well developed for the two-dimensional case and even though some of the properties of the $2 \mathrm{D}$ curves, such as the property of shrinking to a point under curvature flow, do not hold in the 3D case, the main part of the theories remains valid and works well for segmentation of $3 \mathrm{D}$ objects.

To use the geometric flows for image segmentation, the evolution of the curve or surface has to depend on external properties determined by the image features. A classical speed function to segment gray scale images is based on the gradient of the images and goes to zero when the surface approaches an edge [21].

A general flow for a $3 \mathrm{D}$ closed surface can be described as

$\frac{\partial \mathbf{S}}{\partial t}=(F+\kappa) \mathbf{N}$,

where $F$ is an image-based speed function which in our case is dependent on the diffusion image, $\kappa$ is the mean curvature of the surface, $\mathbf{S}$ defines the surface, $\mathbf{N}$ is the normal to the surface and $t$ is the time.

To solve this time dependent PDE we use the level set method, introduced by Osher and Sethian [22], where the evolving surface is considered as a constant level set of an embedding function. This leads to a numerically stable algorithm that easily handles topology changes of the evolving surface. The embedding function is called the level set 
function, $\phi(t)$, of the evolving surface. It has been shown by Osher and Sethian [22] that the evolution of the zero level set coincide with the evolution of $S(t)$. Thus, the evolution of the level set function is described by

$\frac{\partial \phi}{\partial t}=(F+\kappa)|\nabla \phi|$

Image segmentation using region-based statistics have been presented in several early papers [23-26]. Our method is based on a model that was introduced a few years later, the geodesic active region model by Paragios and Deriche [18]. The key hypothesis is that the image is composed of homogeneous regions and that the probability of a pixel belonging to a certain region can be determined from the intensity histogram.

In our case, the probability of a voxel belonging to a certain region is determined by the tensor similarity between a voxel and the most representative tensor, computed according to (3) and (5), for that region. Every voxel in the vicinity of the evolving surfaces is then associated to a region by computing the similarity between the tensor in that voxel and the representative tensors of each level set. The similarity measure that we use for comparing the tensors is the integrated similarity presented in (2).

The similarity measure is the percentage of the common diffusion each tensor has with the different regions. This percentage can be considered as a probability measure of a voxel belonging to a certain region. A region-based force can then be defined according the theories of Paragios et al. [18]:

$F_{i}=-\log \left(\frac{\operatorname{IS}\left(\mathbf{D}, \mathbf{D}_{\mathbf{t y p}, \mathbf{i}}\right)}{\max \left(\operatorname{IS}\left(\mathbf{D}, \mathbf{D}_{\mathbf{t y p}, \mathbf{j} \neq \mathbf{i}))}\right)\right.}\right.$,

where IS is the integrated similarity described in (2). $\mathbf{D}_{\text {typ, } \mathbf{i}}$ is the most representative tensor associated with the level set, $\phi_{i}$ and is computed according to (5). It is continuously recalculated as the surface evolves. $F_{i}$ grows the surface, $S_{i}$, in the direction of the voxels of diffusion most similar to $\mathbf{D}_{\text {typ,i }}$. This choice of representative tensor instead of a mean tensor is important when dealing with tensors that are neither clearly anisotropic nor clearly isotropic. When computing the mean of the tensors within for example the thalamus the mean tensor will develop towards an isotropic tensor.

\subsection{Coupling forces}

When propagating several curves, overlapping can occur. To avoid this, a constraint has to be applied. This is done by adding an artificial force in the direction of the normal, to the corresponding level set motion equations. The force will penalize voxels which have been attributed to more than one region. If necessary, voxels that have not yet been labelled will also be penalized to force each voxel to belong to a region. The coupling forces for each surface, $H_{i}$, are defined as in [18]:

$H_{i}\left(j, \phi_{j}(s)\right)=\frac{1}{N-1} \begin{cases}+1 & \text { if } \phi_{j}(s)>a, \\ -\frac{1}{\tan (1)} \tan \left(\phi_{j}(s) / a\right) & \text { if }\left|\phi_{j}(s)\right| \leqslant a .\end{cases}$

Here, $a$ is a parameter to decide within which distance the coupling force shall act.

\subsection{Final evolution}

Each one of our surfaces, $i$, is now evolving according to:

$\frac{\partial S_{i}}{\partial t}=\left(F_{i}+\kappa_{i}+H_{i}\right) \vec{N}$,

where $F_{i}$ is the region-based force (11), $\kappa_{i}$ is the mean curvature and $H_{i}$ is the coupling force.

\section{Material and methods}

\subsection{Implementation details and parameters}

The method has been implemented in Matlab 6.1 (The MathWorks, Inc.) except for the reinitialization of the signed distance function, which has been implemented in $C$ and compiled with the mexlibrary.

\subsubsection{Convergence}

The evolution of the surfaces is automatically stopped when the zero level set only have moved insignificantly after 10 iterations.

\subsubsection{Preserving the signed distance function}

The evolving surface is considered as the zero level set of its level set function. Due to local dependence of the propagation speed the evolution of the other level sets differs from the zero level set. This could create irregularities that would deform the level set function. A correct level set function is 
crucial for a correct and smooth evolution of the surface, since both computation of normals and curvatures are directly dependent on it. Therefore, a reinitialization of the level set function is made regularly as it is usually performed in the level set literature. See $[27,28]$ for more information concerning this issue. It is implemented using the fast marching method to solve the Eikonal equation (PDE) [29]:

$|\nabla \phi|=1$.

\subsubsection{Weighting the speed terms}

The diffusion dependent, $F_{i}$, and the curvature dependent, $\kappa_{i}$, speed for each surface $S_{i}$, are not always of the same order. For a satisfactory regularization without inhibiting the front propagation it is therefore important to set the weighting factor between them correctly. Hence, we have the following relation:

$\frac{\partial S_{i}}{\partial t}=\left(\alpha F_{i}+\beta \kappa_{i}+\gamma H_{i}\right) \vec{N}$,

where $\alpha, \beta, \gamma$ are weighting parameters.

Finally, the level set function has the following evolution:

$\frac{\partial \phi_{i}}{\partial t}=\left(\alpha F_{i}+\beta \kappa_{i}+\gamma H_{i}\right)\left|\nabla \phi_{i}\right|$

The parameters have been determined empirically and are set to $\alpha=10, \beta=1$ and $\gamma=1$ for all experiments.

\subsection{Synthetic and real data set}

The method has been tested on synthetic data and on diffusion data from two healthy volunteers.

\subsubsection{Synthetic data set}

The method was first validated on a synthetic tensor volume containing regions of tensors with slightly different diffusion properties. Values from different regions of the thalamus were used. These tensors were then placed in six regions in a $3 \mathrm{D}$ volume and Rician noise was added [30]. A cut of a slice through the regions can be seen in Fig 1. To initialize the surfaces we ran a $k$-means clustering algorithm as in [6] and the center points of the obtained clusters were then used as initialization points.

The algorithm was then applied to the tensors fields with a Rician ratio (SNR) of 32 . With a lower

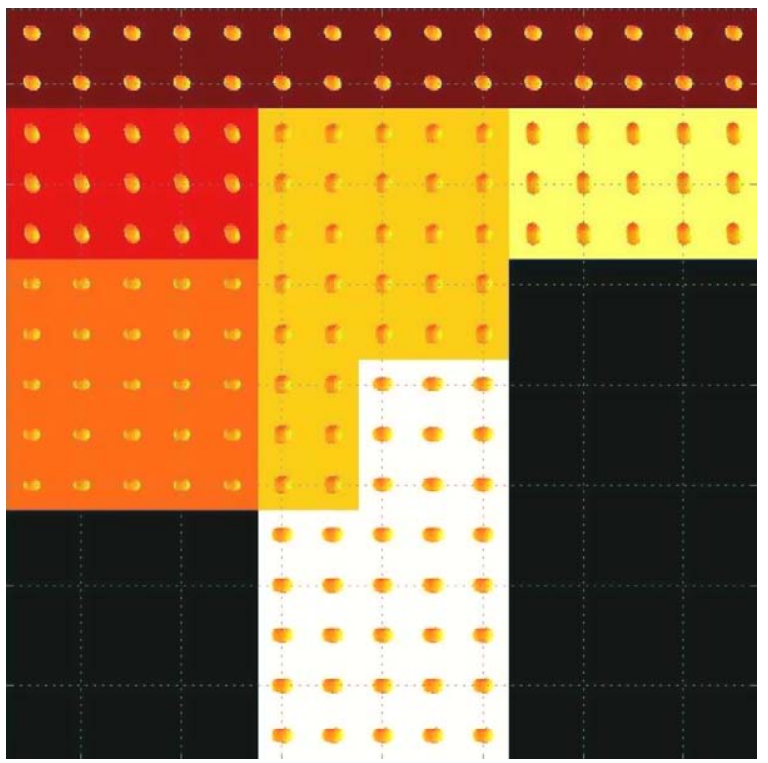

Fig. 1. A cut of the synthetic tensor field used to test the segmentation method. The form of the tensors can be seen in each voxel displayed on a color map representing the anisotropy and principal direction [31].

SNR such similar regions cannot be distinguished so for segmentation of the thalamic nuclei we will clearly need good imaging acquisitions with a reasonably good SNR.

\subsubsection{Real data set}

The images were obtained with a single shot EPI sequence on a $3 T$ Intera scanner from Philipps. Six diffusion weighted images and one without diffusion weighting were acquired. TR and TE were, respectively, 4858 and $78 \mathrm{~ms}$ and $b$ value was set to $1000 \mathrm{~mm} / \mathrm{s}^{2}$. We acquired 24 axial slices in a 256 by 201 matrix that was interpolated to a 256 by 256 matrix, covering the region of the deep cerebral nuclei. The voxel size was $1.0 \mathrm{~mm}$ by $1.0 \mathrm{~mm}$ with a slice thickness of $2.0 \mathrm{~mm}$ without gap. The data were again interpolated to $1.0 \mathrm{~mm}$ by $1.0 \mathrm{~mm}$ by $1.0 \mathrm{~mm}$.

Informed consent was obtained in accordance with institutional guidelines for all of the volunteers.

To validate the results, the segmented thalamus was defined according to the plane crossing the anterior $(\mathrm{AC})$ and the posterior (PC) commissures (AC-PC referential) and co-registered with digital images of the Schaltenbrand and Wahren stereotactic atlas (SWSA) [32]. 


\section{Results}

In Fig. 2 the regions have been segmented on the synthetic tensor field without any noise added. The results are compared with the $k$-means algorithm [6]. We see that our method manages better to segment regions that are more elongated. The $k$ means clustering algorithm weights the distance between the tensors as well as the similarity between them and when they are far apart they get more easily attributed to an other region. If the algorithm puts more weight to the similarity than the spatial distance the clusters becomes less centered and nonconnected clusters can appear. In Fig. 2 the same segmentations have been made on a field with a $\mathrm{SNR}=32$.

\subsection{Segmentation of the thalamus}

For the segmentation of the thalamus, a bloc of the complete images containing the desired structure was selected. With an a priori knowledge of brain anatomy several surfaces were initiated manually in the different structures by looking on color maps [31]. A typical choice of initial surfaces can be seen in Fig. 3. The high resolution of our images makes a strong distinction between the anterior and posterior regions of the thalamus. We therefore initiate two surfaces for the thalamus, one surface for the anterior part and one surface for the posterior part. The posterior part itself is one of the nuclei, the pulvinar nuclei.

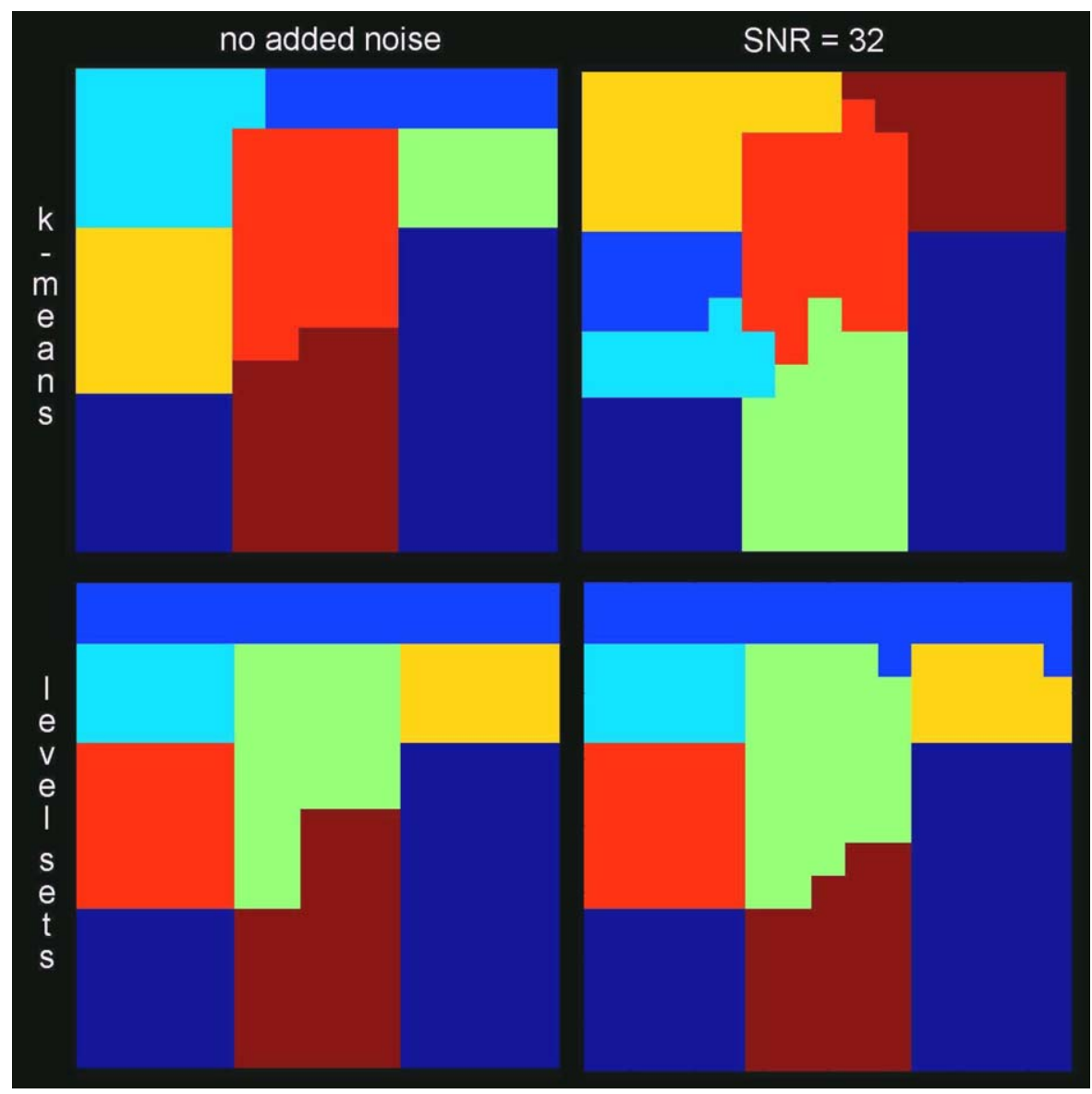

Fig. 2. A cut of the segmentation result on a synthetic field. First line contains the results from the $k$-means algorithm [6]. The second line is the results with the method presented in this chapter. First column shows results without any noise added. Second column with SNR $=$ 32. The level set methods results in a better segmentation for elongated structures and when noise is present and also seems more robust to noise. 


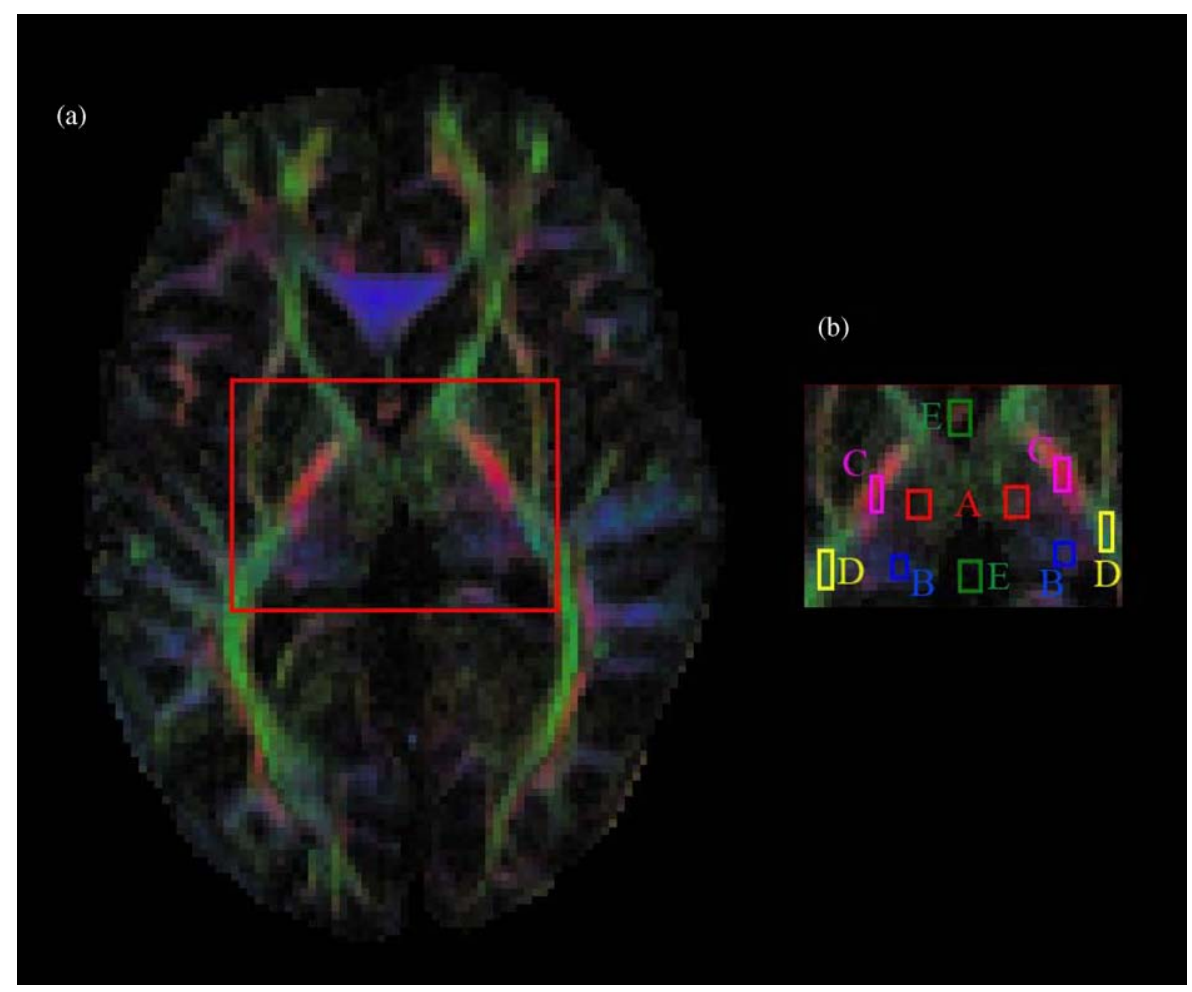

Fig. 3. Placing of the initial surfaces for the segmentation of the thalamus. (a) Color map of a horizontal section of a tensor field. (b) The selected cut where the segmentation algorithm is run with the initial surfaces. They aim to segment the following structures: (A) thalamus anterior, (B) thalamus posterior (pulvinar nuclei), (C) capsula interna, crus posterius (cortico spinal tract), (D) radiato optica and fasciculus longitudinalis superior, and (E) the third ventricule.

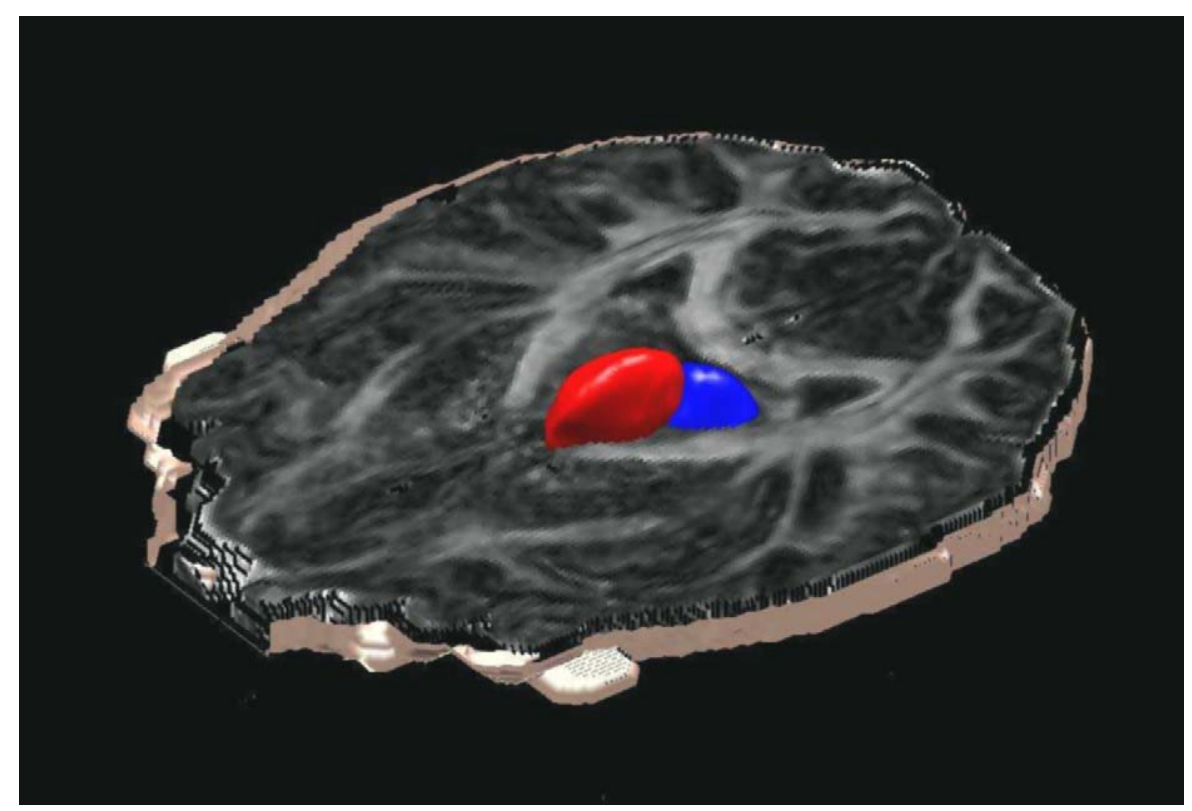

Fig. 4. The segmentation of the thalamus displayed as a 3D surface on a horizontal cut of the fractional anisotropy map. The anterior (red surface) and posterior part (pulvinar nuclei, blue surface) are segmented separately. 

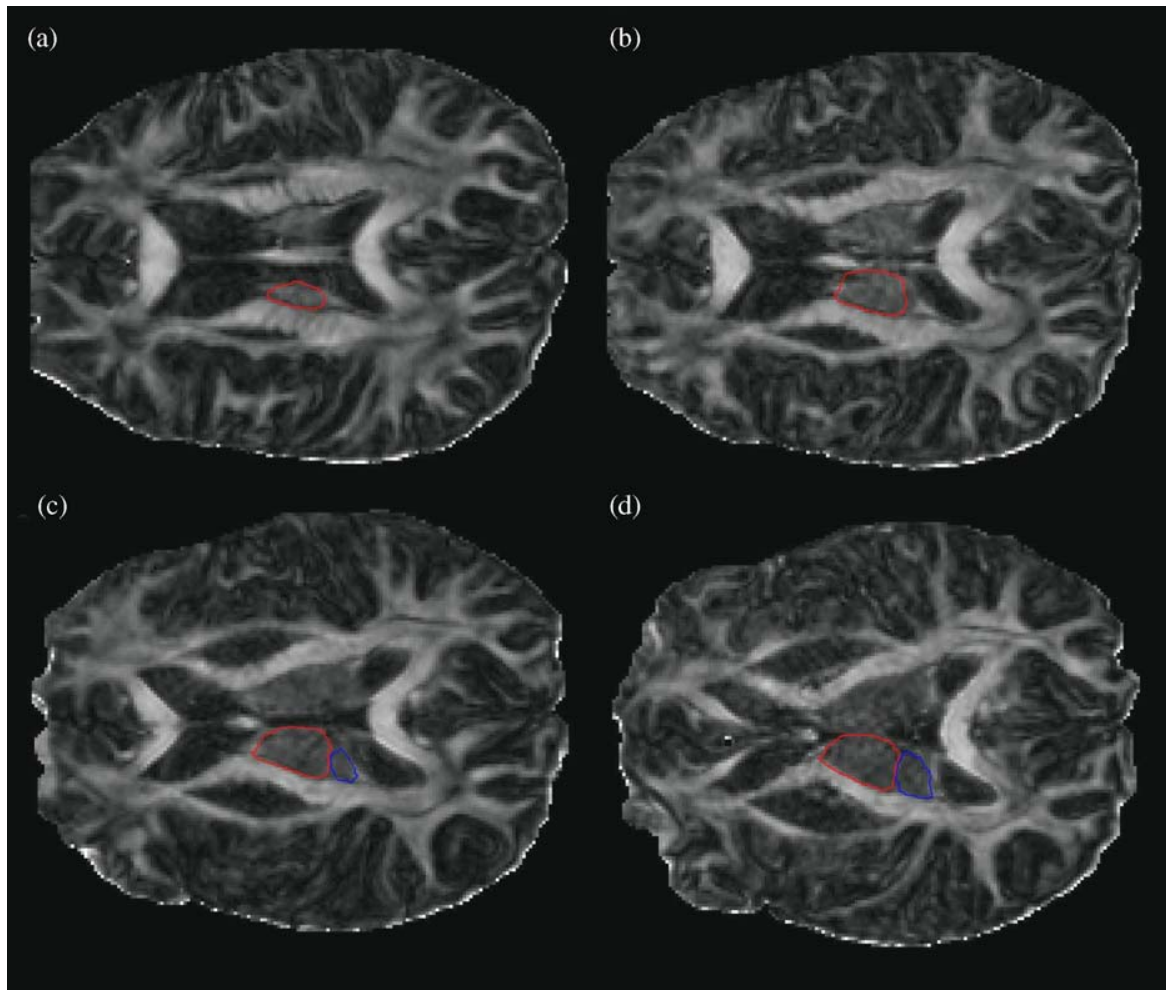

Fig. 5. The segmentation of the thalamus displayed on horizontal cuts of the fractional anisotropy map. The anterior (red surface) and posterior part (pulvinar nuclei, blue surface) are segmented separately.
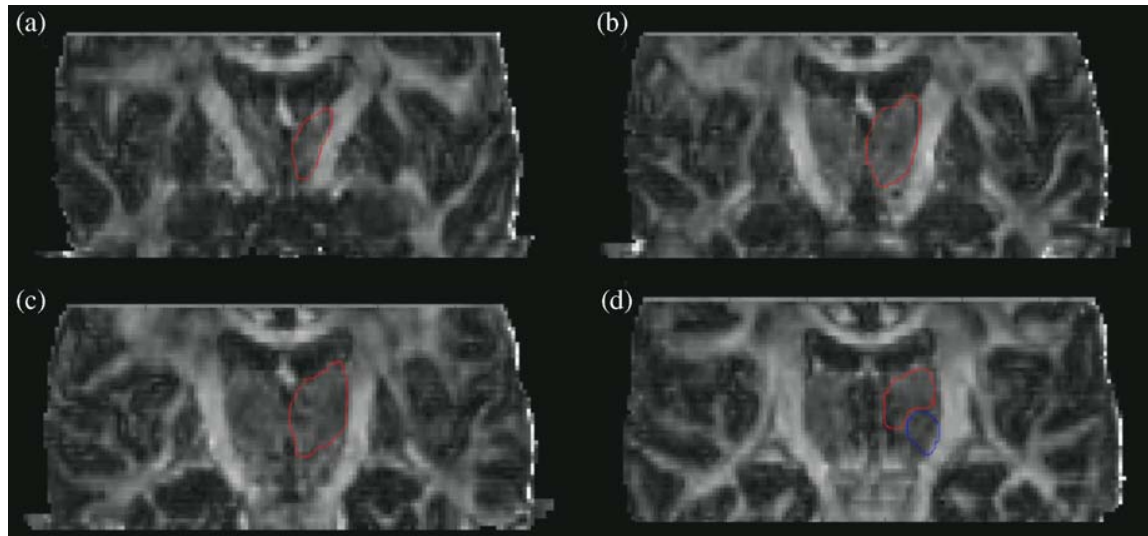

(d)
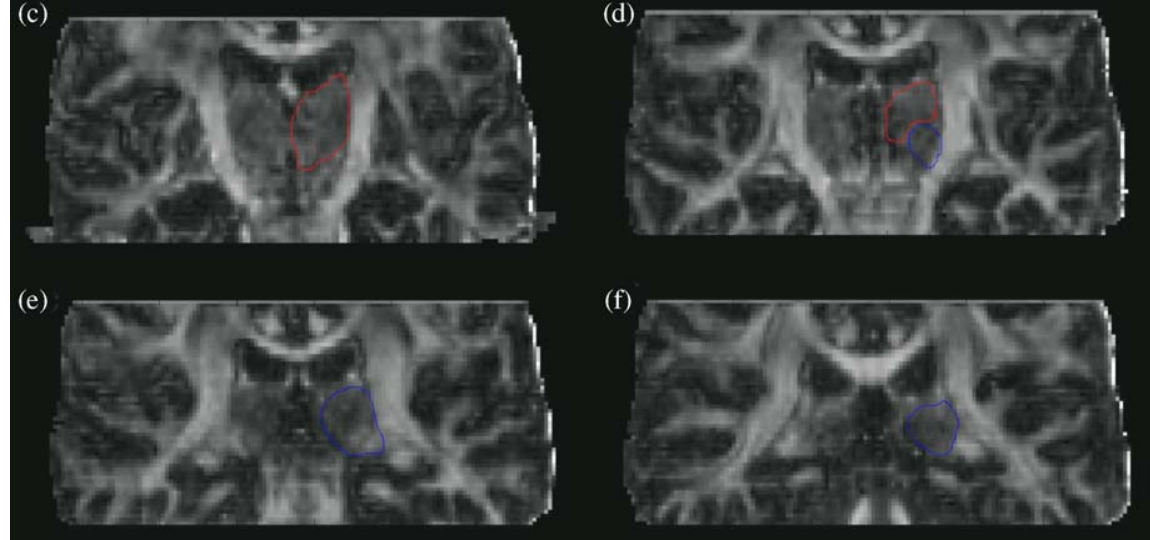

Fig. 6. The segmentation of the thalamus displayed on coronal cuts of the fractional anisotropy map. The anterior (red surface) and posterior part (pulvinar nuclei, blue surface) are segmented separately. 


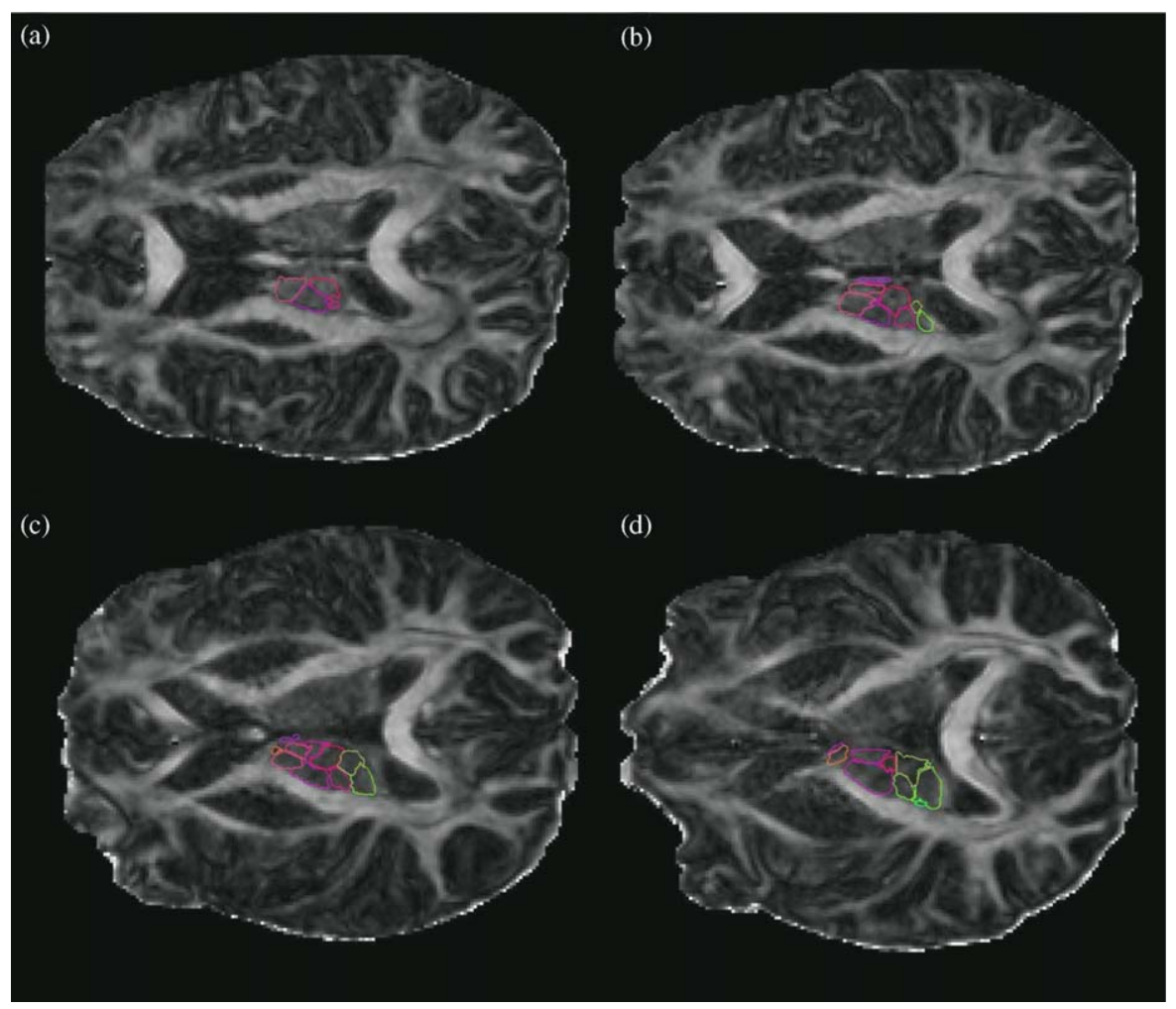

Fig. 7. The segmentation of the thalamic nuclei displayed on horizontal cuts of the fractional anisotropy. Segmentation is made in 14 parts. The color of the contours represent the direction of the principal direction of the representative tensor of the region.

The thalamus has been segmented on two different subjects. The results for one of the subjects can be seen in Figs. 4-6.

Taking the segmentation one step further we have segmented the thalamic nuclei. For this the thalamus segmentation is used as a mask. The surfaces are initialized by running the $k$-means algorithm by Wiegell et al. [6]. The center of each cluster was the used as initial points for the surfaces.

The results can be seen in Figs. 7-9. The colors of the contours represent the direction of the principal direction of the representative tensor of the region. We see that the color varies very slightly between the different segmented nuclei but we can see a significant difference between the anterior and posterior nuclei. No significant difference can be seen in the values of fractional anisotropy between the nuclei, the fractional anisotropy varies between 0.26 and 0.33 with a standard deviation around 0.7 . For the thalamus as a whole these values are 0.27 and 0.7 . From the result the different nuclei have been identified by an expert.

Fig. 10 shows the correlation between axial slices of the segmented thalamus performed 2 and $7 \mathrm{~mm}$ above AC-PC plane and co-registered with the slices of the SWSA performed at the same levels. $\mathrm{Pu}$ : pulvinar, Ce: nucleus centralis, $\mathrm{M}$ : nucleus medialis, A: nucleus anterior, Lp: nucleus lateropolaris : $\mathrm{Zc}+\mathrm{Zim}+\mathrm{Zo}$ : nucleus Zentrooralis caudalis, intermedius and oralis, Tmth: tractus mamillothalamicus, Vc: nucleus ventrocaudalis, Vim: nucleus ventralis intermedius, Vo: nucleus ventrooralis.

\section{Discussion and conclusion}

We have presented a new method for segmenting DT-MRI by using diffusive similarity. The similarity is used to define a region-based force that drives a set of coupled 3D surfaces towards the optimal segmentation. The method is implemented using the level set method and has been applied to DT-MRI of white matter as well as gray matter. The method has shown to be capable of distinguishing and separating regions with only very subtle differences in diffusion such as the difference between the thalamic nuclei.

The method we propose to segment the thalamus and the thalamic nuclei is a continuation of our 

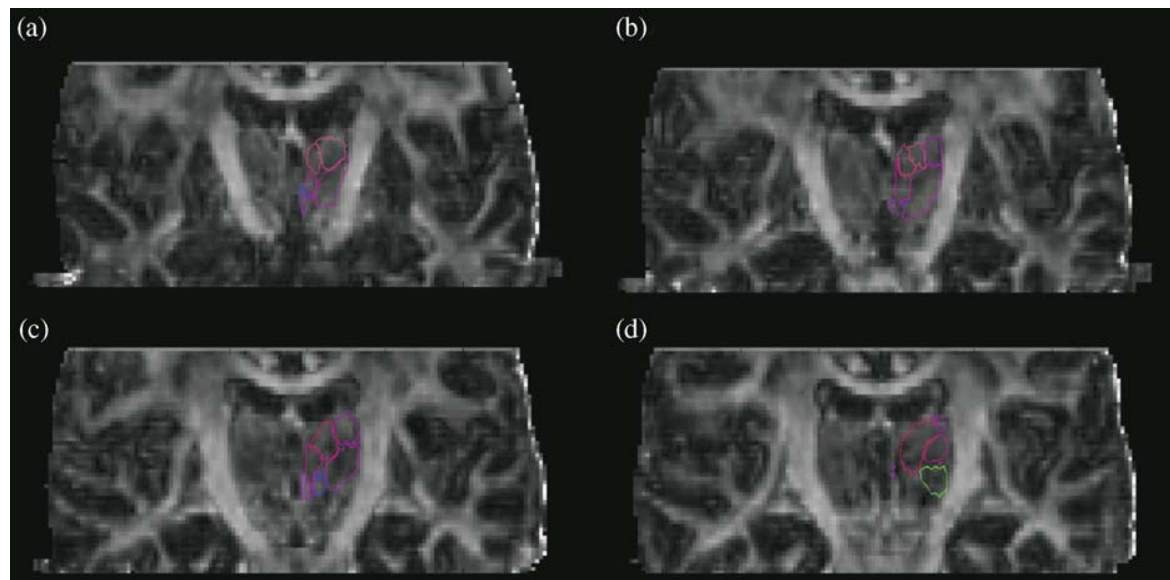

(d)

(e)

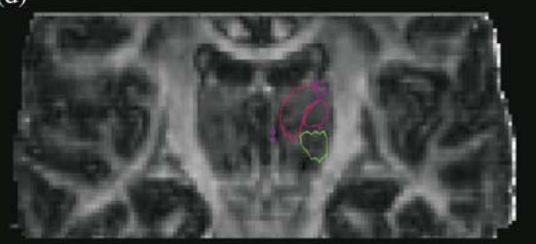

(f)
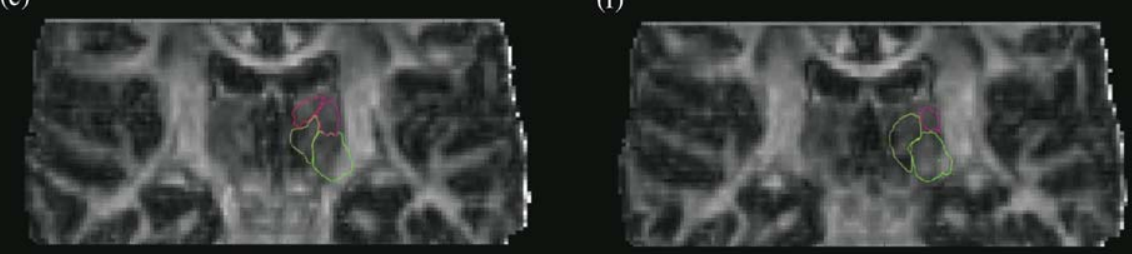

(g)

(h)
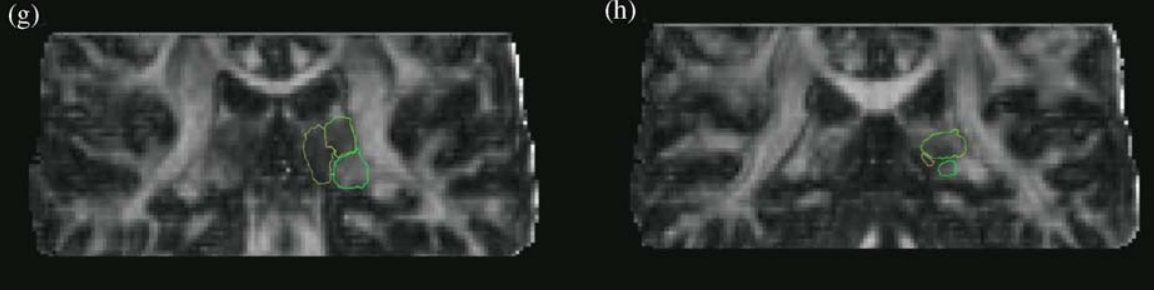

Fig. 8. The segmentation of the thalamic nuclei displayed on coronal cuts of the fractional anisotropy. Segmentation is made in 14 parts. The color of the contours represent the direction of the principal direction of the representative tensor of the region.

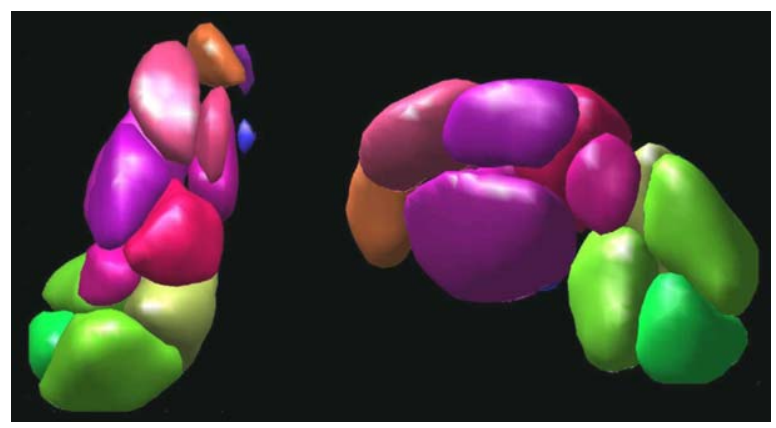

Fig. 9. The segmentation of the thalamic nuclei seen in 3D. Segmentation is made in 14 parts. The color of the contours represent the direction of the principal direction of the representative tensor of the region.

work on fiber tract segmentation. It distinguishes itself on three main points. The first main difference of our approach is the similarity measure we use. It is capable of detecting very subtle changes between tensors. When segmenting gray matter structures these subtle differences are more important than for white matter segmentation.

The second important point in our work is the choice of the most representative tensor of each level set. The approach proposed by Wang et al. [12] is to compute a mean tensor for each region. For regions with high inter-resemblance, the mean tensor has a tendency of developing towards an isotropic tensor and all regions will then be associated to similar tensors. This can be avoided by using a method proposed by Jones et al. [20] that was originally used to find the tensor image that best represented a whole set of images. We have transformed this method to find the tensor that best represents the set of tensors contained within a surface. The third important difference is the use of several coupled level sets that each represent a region we wish to segment. When segmenting structures with such complex architecture as the 


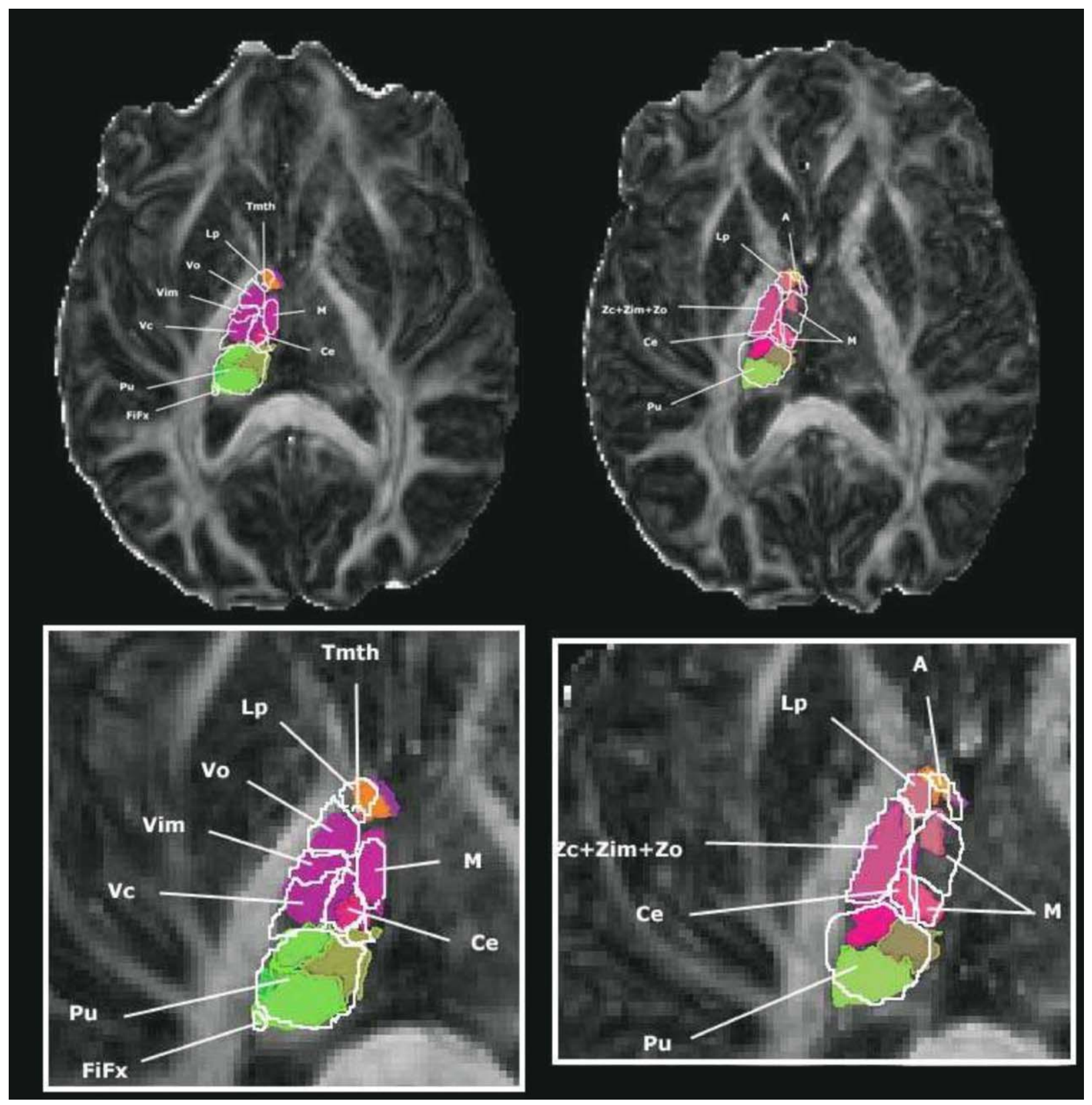

Fig. 10. The segmentation of the thalamic nuclei co-registered with SWSA.

brain, simply separating foreground and background is mostly insufficient. Using such a simplification leads to either an over- or under segmentation of the desired structure. Using several coupled level set that each represents a region, increases robustness of the method.

When comparing results with the $k$-means algorithm we can state that the advantages of the $k$ means algorithm are that it is fast and does not require any pre-initialization. The advantages of our methods are a higher flexibility regarding the shape of the nuclei and that it diminishes the influence of noise due to the self-regularization of the surfaces.

The method generates good results for segmentation of the thalamus. As for the thalamic nuclei, the validation shows that the level set method applied on DT-MRI of the thalamus provides an overall consistent segmentation of the thalamic nuclei 
related to their anatomical position defined by the SWSA. For some nuclei (pulvinar), it was even able to show subdivisions within the structure, already described in the SWSA. For others (nucleus ventralis or zentrooralis), it could not recognize the caudal, intermediate and oral portions of the nucleus. Comprehension of these observations is beyond the scope of this paper and should be better analyzed in a further study.

\section{Acknowledgments}

This work is supported by the Swiss National Science Foundation grant number 2153-066943.01.

\section{References}

[1] C. Beaulieu, The basis of anisotropic water diffusion in the nervous system - a technical review, NMR Biomed. 15 (7-8) (2002) 435-455.

[2] P. Hagmann, J.P. Thiran, L. Jonasson, P. Vandergheynst, S. Clarke, R. Meuli, DTI mapping of human brain connectivity: statistical fibre tracking and virtual dissection, NeuroImage 19 (3) (2003) 545-554.

[3] T.E. Conturo, N.F. Lori, T.S. Cull, E. Akbudak, A.Z. Snyder, J.S. Shimony, R.C. McKinstry, H. Burton, M.E. Raichle, Tracking neuronal fiber pathways in the living human brain, Proc. Nat. Acad. Sci. USA 96 (18) (1999) 10422-10427.

[4] B.C. Vemuri, R.M. Chen, Y.T. McGraw, Z. Wang, T. Mareci, Fiber tract mapping from diffusion tensor MRI, in: Proceedings of the IEEE Workshop on Variational and Level Set Methods in Computer Vision, 2001, pp. 81-88.

[5] P.J. Basser, S. Pajevic, C. Pierpaoli, J. Duda, A. Aldroubi, In vivo fiber tractography using DT-MRI data, Magn. Reson. Med. 44 (4) (2000) 625-632.

[6] M.R. Wiegell, D.S. Tuch, H.B.W. Larsson, V.J. Wedeen, Automatic segmentation of thalamic nuclei from diffusion tensor magnetic resonance imaging, NeuroImage 19 (2003) 391-401.

[7] T.E. Behrens, H. Johansen-Berg, M.W. Woolrich, S.M. Smith, C.A. Wheeler-Kingshott, P.A. Boulby, G.J. Barker, E.L. Sillery, K. Sheehan, O. Ciccarelli, A.J. Thompson, J.M. Brady, P.M. Matthews, Non-invasive mapping of connections between human thalamus and cortex using diffusion imaging, Nat. Neurosci. 6 (7) (2003) 750-757.

[8] C.R. Tench, P.S. Morgan, M. Wilson, L.D. Blumhardt, White matter mapping using diffusion tensor MRI, Magn. Reson. Med. 47 (5) (2002) 967-972.

[9] L. Jonasson, P. Hagmann, X. Bresson, R. Meuli, O. Cuisenaire, J.-Ph. Thiran, White matter mapping in DTMRI using geometric flows, in: Computer Aided Systems Theory-Eurocast 2003, Lecture Notes in Computer Science, vol. 2809, Springer, Berlin, 2003, pp. 585-595.

[10] L. Jonasson, X. Bresson, P. Hagmann, R. Meuli, O. Cuisenaire, J.-Ph. Thiran, White matter fiber tract segmentation in DT-MRI using geometric flows, Med. Image Anal. 9 (3) (2005) 223-236.
[11] L. Zhukov, K. Museth, D. Breen, R. Whitaker, A. Barr, Level set modelling and segmentation of DT-MRI brain data, J. Electron. Imaging 12 (1) (2003) 125-133.

[12] Z. Wang, B. Vemuri, Tensor field segmentation using region based active contour model, in: ECCV 2004, Lecture Notes in Computer Science, vol. 3024, Springer, Berlin, 2004, pp. 304-315.

[13] C. Feddern, J. Weickert, B. Burgeth, Level-set methods for tensor valued images, in: Proceedings of the Second IEEE Workshop on Variational, Geometric and Level Set Methods in Computer Vision, 2003, pp. 65-72.

[14] M. Rousson, C. Lenglet, R. Deriche, Level set and region based surface propagation for diffusion tensor MRI segmentation, in: CVAMIA and MMBIA, 2004.

[15] C. Lenglet, M. Rousson, R. Deriche, O. Faugeras, Inferring white matter geometry from diffusion tensor MRI: application to connectivity mapping, in: Pajdla and Matas, Prague, Czech Republic, Springer, Berlin, 2004.

[16] Z. Wang, B. Vemuri, An affine invariant tensor dissimilarity measure and its applications to tensor-valued image segmentation, in: Computer Vision and Pattern Recognition, 2004, CVPR 2004, Proceedings of the 2004 IEEE Computer Society Conference, 2004, pp. 228-233.

[17] C. Lenglet, M. Rousson, R. Deriche, Segmentation of $3 \mathrm{~d}$ probability density fields by surface evolution: application to diffusion MRI, in: Proceedings of the Seventh International Conference on Medical Image Computing and Computer Assisted Intervention, Saint-Malo, France, September 2004.

[18] N. Paragios, R. Deriche, Geodesic active regions: a new paradigm to deal with frame partition problems in computer vision, J. Visual Commun. Image Representation March/ June (2003).

[19] D. Alexander, J. Gee, R. Bajcsy, Similarity measures for matching diffusion tensor images, in: Proceedings BMCV'99, 1999.

[20] D.K. Jones, L.D. Griffin, D.C. Alexander, M. Catani, M.A. Horsfield, R. Howard, S.C. Williams, Spatial normalization and averaging of diffusion tensor MRI data sets, NeuroImage 17 (2002) 592-617.

[21] V. Caselles, R. Kimmel, G. Sapiro, Geodesic active contours, Int. J. Comput. Vision 22 (1997) 61-79.

[22] S. Osher, J.A. Sethian, Fronts propagating with curvaturedependent speed: algorithms based on Hamilton-Jacobi formulations, J. Comput. Phys. 79 (1988) 12-49.

[23] A. Yezzi, A. Tsai, A. Willsky, A curve evolution approach to smoothing and segmentation using the mumford-shah functional, in: IEEE Conference on Computer Vision and Pattern Recognition, 2000.

[24] A. Yezzi, A. Tsai, A. Willsky, A statistical approach to snakes for bimodal and trimodal imagery, in: Seventh IEEE International Conference on Computer Vision, 1999.

[25] J. Zhu, J.A. Sethian, Projection methods coupled to level set interface techniques, J. Comput. Phys. 102 (1992) 128-138.

[26] T.F. Chan, L.A. Vese, Active contours without edges, IEEE Trans. Image Process. 10 (2) (2001) 266-277.

[27] M. Sussman, P. Smereka, S. Osher, A level set approach for computing solutions to incompressible two-phase flow, J. Comput. Phys. 114 (1994) 146-159.

[28] D. Peng, B. Merriman, S. Osher, H. Zhao, M. Kang, A pdebased fast local level set method, J. Comput. Phys. 155 (2) (1999) 410-438. 
[29] M. Sussman, E. Fatemi, An efficient, interface preserving level set re-distancing algorithm and its application to incompressible fluid flow, SIAM J. Sci. Comput. 20 (4) (1999) 1165-1191.

[30] J.D. Tournier, F. Calamante, M.D. King, D.G. Gadian, A. Connelly, Limitations and requirements of diffusion tensor fiber tracking: an assessment using simulations, Magn. Reson. Med. 47 (4) (2002) 701-708.
[31] S. Pajevic, C. Pierpaoli, Color schemes to represent the orientation of anisotropic tissues from diffusion tensor data: application to white matter fiber tract mapping in the human brain, Magn. Reson. Med. 42 (3) (1999) 526-540.

[32] G. Schaltenbrand, W. Wahren, Atlas for Stereotaxy of the Human Brain, 1977. 